\title{
Peridiagnostic and cascade cancer genetic testing
}

Nora Pashayan ${ }^{1}$, Clare Turnbull2,3

${ }^{1}$ Department of Applied Health Research, Institute of Epidemiology and Healthcare, University College London, UK

${ }^{2}$ Division of Genetics and Epidemiology, Institute of Cancer Research, UK

${ }^{3}$ Genomics England, Queen Mary University of London, UK

\section{Corresponding author:}

Nora Pashayan, University College London, Department of Applied Health

Research, 1-19 Torrington Place, London, WC1E 7HB, UK

e-mail: $\underline{n . p a s h a y a n @ u c l . a c . u k ~}$

Words: 935 


\section{Summary}

Identification of cancer susceptibility genetic variants could be improved by peridiagnostic cancer genetic testing and cascade testing of the relatives with the active involvement of the medical team in informing the relatives and offering pre-test telephone genetic counselling. 
It is over 20 years since identification of high penetrance cancer susceptibility genes such as BRCA1, BRCA2, MLH1 and MSH2. Clinical testing for these genes in individuals with a priori high risk of carrying pathogenic variants (mutation) has been widely implemented on the basis of reliable evidence that the risk and the impact of the associated cancers can be mitigated via specific clinical interventions, including risk-reducing medications and surgery, and enhanced surveillance. Based on personal or family history suggestive of inherited cancer susceptibility, 'full mutational screening' of the relevant genes is offered to patients deemed at elevated likelihood of carrying a pathogenic variant in one of these genes, typically through clinical genetics services or oncology. On identification of a pathogenic variant in the 'index proband', targeted testing for this specific variant is typically offered to family members, cascading out through branches of the family in a stepwise manner.

There is ongoing scrutiny within clinical genetics and oncology communities regarding optimal approaches to identify index cancer susceptibility gene pathogenic variant carriers (CSG-PV-Cs) and the relative merits of (a) genetic testing of high risk individuals based on family and/or personal history of cancer, (b) peridiagnostic genetic testing amongst (unselected) patients with specific cancer types (or even any cancer), (c) testing of the general unselected population. Alongside these debates are often discussions around how to improve our systems for cascade testing from the index proband, that is how to get maximum 'bang for buck' for each new proband identified. Writing in the Journal of Clinical Oncology, Offit et $\mathrm{al}^{1}$ and Frey et $\mathrm{al}^{2}$, explored how to approach the cascade testing of patients newly diagnosed with cancer.

Offit et al $^{1}$ modelled various scenarios of peri-diagnostic cancer genetic testing combined with cascade testing in first-, second-, and third-degree relatives (FDR, SDR, TDR, respectively). They estimated that in the USA across 18 high penetrance cancer susceptibility genes there would be a total of 3.9 million cancer susceptibility gene pathogenic variant carriers (CSG-PV-Cs). They modelled the time interval to detect the estimated 3.9 million CSG-PV-Cs by varying (i) the proportion of patients with cancer tested for CSGs (range, $7.5 \%$ to $75 \%$ ), (ii) the proportion of patients in whom a CSG-PV is detected (5\% to 15\%), (iii) the proportion of FDR, SDR, TDR tested for the familial pathogenic variants $(0 \%$ to $75 \%$ ). Assuming a $7 \%$ prevalence of pathogenic variants across cancer types, an 
average family sibship of three per generation, and $15 \%$ of the incident cancer patients tested each year, Offit et al showed that detection of all 3.9 million CSG-PVCs would require 46.2 years if $10 \%$ of all FDR, SDR, and TDRs were tested but could be achieved in as little as 9.9 years if 70\% FDR, SDR, and TDRs were tested. Extrapolation from their outputs should be caveated by the assumptions inherent to their model, which include (i) the population mutation carrier rate (3.9 million) is fixed whilst the mutation detection rate in cancer patients can vary, (ii) randomness of cancer patients selected for CSG testing, (iii) a steady state in space and time of CSG-PV-Cs, (iv) consistency of sibship size between and within generations, (v) absence of complex families (e.g. half-siblings), and (vi) absence of relatedness between cancer patients.

Frey et $\mathrm{al}^{2}$ studied the feasibility of offering "Facilitated Cascade Genetic Testing Pathway" to 114 at-risk relatives (ARRs) of 30 CSG-PV probands diagnosed with a hereditary cancer syndrome within the past year. This pathway comprised clinicallysupervised pedigree review with the proband to identify all ARRs, active contact of ARRs by the genetic counsellor, telephone counselling for CSG testing and sampling via postal saliva kit. Among the 114 ARRs, 93\% were successfully contacted, and $58 \%$ completed genetic testing. At 6-month follow-up, among 27 ARRs identified with pathogenic variant, seven had undergone cancer surveillance interventions and 4 had cancer risk-reducing surgery. The test uptake rate of $58 \%$ is similar to previous studies of clinician-led cascading ${ }^{3}$. This rate is significantly higher than the $\sim 30 \%$ rate observed across clinical practice, in which cascading is typically less actively managed and more patient-led.

These two studies serve to highlight the importance of cascade testing as an impactful and cost-efficient process by which to identify additional CSG-PV-Cs downstream of identification of any index proband. However, the genetic testing rate of $58 \%$ attained in Frey et $\mathrm{al}^{2}$ is sobering, given that their model is clinician-intensive and their genetic testing did not require negotiation for reimbursement. This indicates that any inferences from Offit et $\mathrm{al}^{1}$, should utilise the more conservative estimates of cascading, namely $25-50 \%$. Nevertheless, the excess reliance on cascading may be misplaced and the onus likely must remain on ascertainment of index CSG-PV-Cs. 
Overall ascertainment of CSG-PV-Cs remains poor, even in countries with well evolved genetics services. Genetic testing of high-risk individuals based on family history would detect less than $50 \%$ of CSG-PV-Cs. Population-wide testing could potentially identify all CSG-PV-Cs. However, resource implications, uptake of the test and the subsequent interventions, equitable accessibility, feasibility of implementation, psychological distress, overdiagnosis and side-effects of subsequent clinical interventions remain important considerations. For example, the US Preventive Services Task Force recommended against population-based testing for pathogenic variants in BRCA genes on the grounds that the potential harms of genetic testing and subsequent interventions outweigh the benefits ${ }^{5}$. Genetic testing of patients with cancer diagnosis remains a plausible alternative. Who (e.g. age, cancer type), when (e.g. at time of diagnosis, or after treatment), and what test to offer remain to be explored. New programmes are urgently required to evaluate and compare the effectiveness, cost-effectiveness, and feasibility of extension of genetic testing via familial cancer clinics, mainstream peri-diagnostic testing in oncology and population-based programmes. 
Competing interests

NP and CT have none to declare 


\section{References}

1. Offit, K. et al. Cascading after peridiagnostic cancer genetic testing: an alternative to population-based screening. J Clin Oncol JCO1902010 (2020). doi: 10.1200/JCO.19.02010.

2. Frey, M.K. et al. Prospective Feasibility Trial of a Novel Strategy of Facilitated Cascade Genetic Testing Using Telephone Counseling. J Clin Oncol JCO1902005 (2020). doi: 10.1200/JCO.19.02005.

3. Evans, D.G. et al. Comparison of proactive and usual approaches to offering predictive testing for BRCA1/2 mutations in unaffected relatives. Clin Genet 75,124-132 (2009).

4. Møller, P. et al. Genetic epidemiology of BRCA mutations-family history detects less than $50 \%$ of the mutation carriers. Eur $J$ Cancer 43(11), 17131717 (2007). doi:10.1016/j.ejca.2007.04.023.

5. US Preventive Services Task Force. Risk assessment, genetic counselling. And genetic testing for $B R C A$ related cancer. US Preventive services Task Force recommendation statement. JAMA 322(7), 652-665 (2019). doi: 10.1001/jama.2019.10987. 